\title{
RELAÇÕES INTERPESSOAIS NO CENTRO CIRÚRGICO: EQUIPE DE ENFERMAGEM E EQUIPE MÉDICA
}

\author{
INTERPERSONAL RELATIONS IN THE SURGICAL CENTER: NURSING TEAM AND MEDICAL TEAM
}

\section{RELACIONES INTERPERSONALES EN EL CENTRO QUIRÚRGICO: EQUIPO DE ENFERMERÍA Y EQUIPO MÉDICO}

\author{
Anna Maria Oliveira Salimena ${ }^{1}$, Raquel Santos Rosa Peixoto ${ }^{2}$, Silvia Teresa Carvalho Araújo ${ }^{3}$, Marcelo Silva Alves ${ }^{4}$
}

\section{RESUMO}

Objetivo: Compreender significados e desvelar sentidos da equipe de enfermagem em centro cirúrgico nas suas relações interpessoais com a equipe médica. Método: Pesquisa de natureza qualitativa, embasada no referencial teórico, filosófico e metódico na fenomenologia Heideggeriana. Participaram 17 profissionais da equipe de enfermagem de um centro cirúrgico, entre os meses de outubro de 2016 a janeiro de 2017, utilizando a entrevista fenomenológica norteada pela questão: Como você significa as suas relações interpessoais com a equipe médica no seu trabalho?. Dos depoimentos emergiram as estruturas essenciais, a construção do conceito de ser e a unidade de significado, possibilitando desvelar os sentidos dos profissionais. Resultado: Os profissionais de enfermagem significaram as relações interpessoais com a equipe médica como boa, mas com conflitos e divergências. Foram desvelados os sentidos ser-com, impropriedade, inautencidade, ambiguidade e distanciamento. Considerações Finais: Dos sentidos desvelados considera-se imprescindíveis que as relações interpessoais estabelecidas sejam harmônicas, baseadas na empatia, respeito e comunicação eficaz a fim de garantir uma assistência segura ao paciente.

Descritores: Centros Cirúrgicos; Relações Interpessoais; Equipe de Enfermagem.

\begin{abstract}
Objective: To understand and reveal meanings of the nursing team in a surgical center in their interpersonal relationships with the medical team. Method: Research of a qualitative nature, based on the theoretical, philosophical and methodical reference of the Heideggerian phenomenology. A total of 17 professionals of the nursing team of a surgical center participated in the study, which was carried out between the months of October 2016 to January 2017, using the phenomenological interview guided by the following question: What is the significance of your interpersonal relationships with the medical staff in your job?. Essential structures merged from the testimonies: the construction of the concept of being and the unity of meaning making it possible to unveil the senses of professionals. Outcome: Nursing professionals meant interpersonal relationships with the medical team as good, but with conflicts and disagreements. The senses of being-with, impropriety, inauthenticity, ambiguity and distance were unveiled. Final Considerations: From the unveiled senses, it is considered essential that the established interpersonal relations ought to be harmonious, based on empathy, respect and effective communication in order to guarantee a safe assistance to the patient.
\end{abstract}

Descriptors: Surgicenters; InterpersonalRelations; Nursing,Team.

\section{RESUMEN}

Objetivo: Comprender significados y desvelar sentidos del equipo de enfermería en centro quirúrgico en sus relaciones interpersonales con el equipo médico. Método: Investigación de naturaleza cualitativa, basada en el referencial teórico, filosófico y metódico en la fenomenología Heideggeriana. Participaron 17 profesionales del equipo de enfermería de un centro quirúrgico, entre los meses de octubre de 2016 a enero de 2017, utilizando la entrevista fenomenológica orientada por la cuestión: ¿Cómo te refieres a tus relaciones interpersonales con el equipo médico en tu trabajo? De los testimonios surgieron las estructuras esenciales, la construcción del concepto de ser y la unidad de significado posibilitando desvelar los sentidos de los profesionales. Resultado: Los profesionales de enfermería se refirieron a las relaciones interpersonales con el equipo médico como buenas, pero con conflictos y divergencias. Se desvelaron los sentidos ser-con, impropiedad, inautenticidad, ambigüedad y distanciamiento. Consideraciones finales: De los sentidos desvelados se considera imprescindible que las relaciones interpersonales establecidas sean armónicas, basadas en la empatía, el respeto y la comunicación eficaz para garantizar una asistencia segura al paciente. Descriptores: Centros Quirúrgicos; Relaciones interpersonales; Grupo de Enfermería.

${ }^{1}$ Enfermeira. Doutora. Professora do Programa de Pós-graduação Graduação Mestrado em Enfermagem da Faculdade de Enfermagem da Universidade Federal de Juiz de Fora. ${ }^{2}$ Enfermeira. Mestranda do Programa de Pós-graduação Mestrado em Enfermagem da Faculdade de Enfermagem da Universidade Federal de Juiz de Fora. ${ }^{3}$ Enfermeira. Doutora. Professora do Programa de Pós-Graduação Mestrado em Enfermagem da Escola de Enfermagem Anna Nery da Universidade Federal do Rio de Janeiro. ${ }^{4}$ Enfermeiro. Doutor. Professor do Programa de Pós-graduação Graduação Mestrado em Enfermagem da Faculdade de Enfermagem da Universidade Federal de Juiz de Fora. 


\section{INTRODUÇÃO}

O Centro Cirúrgico é considerado um setor complexo e de alto risco tanto pela sua especificidade quanto por ser um setor restrito que impõe à equipe de saúde momentos estressantes ao lidar com aspectos referentes à competência técnica, recursos materiais e relacionamento interpessoal ${ }^{(1)}$. 0 setor é organizado por um conjunto de áreas e instalações, com a finalidade de realizar os procedimentos anestésico-cirúrgicos nas melhores condições possíveis de segurança para o paciente e conforto para a equipe que realiza a assistência $^{(2)}$.

O trabalho é realizado por uma equipe multiprofissional, entre elas a equipe de enfermagem, a equipe cirúrgica, a equipe de anestesiologistas, a equipe da limpeza e, em alguns hospitais, também faz parte a equipe administrativa. Torna-se imprescindível que haja uma atuação harmônica e integrada entre as equipes, para a segurança do cliente e eficiência de todo o serviço ${ }^{(3)}$. Nesse sentido, é fundamental que o ambiente seja saudável, com bom relacionamento entre as equipes de trabalho, visando à segurança e bem-estar dos pacientes, bem como o bom desenvolvimento das atividades propostas ${ }^{(4)}$.

As relações interpessoais podem ser consideradas como instrumentos de trabalho e sua falta ou limitação pode acarretar relações impessoais distantes e conflitantes o que afeta diretamente o cuidado. Para isso é necessário desenvolver habilidades sociais como a comunicação, a resolução de problemas, cooperação, empatia e assertividade, a fim de embasar o processo de interação ${ }^{(5)}$. Desse modo, as relações interpessoais e a presença dessas habilidades são relevantes no desenvolver das relações estabelecidas e atua como facilitador no ambiente de trabalho, auxiliando o trabalho colaborativo, além de promover a satisfação do trabalhador de enfermagem na realização das tarefas em equipe e no tratamento do paciente ${ }^{(6)}$.

Além disso, o conhecimento das relações interpessoais em centro cirúrgico é necessário e essencial para traçar metas a fim de promover a melhoria no relacionamento das equipes e priorizar uma assistência de enfermagem de qualidade $^{(7)}$. Portanto, compreender sobre as relações estabelecidas entre as equipes torna-se primordial, pois os descontentamentos influenciarão na assistência ao paciente. Essa compreensão auxiliará a resolução dos problemas e o atendimento de qualidade ${ }^{(8)}$. Os maiores estressores no trabalho da equipe de enfermagem são os conflitos interpessoais entre enfermeiros e médicos e entre enfermeiros e sua equipe $^{(9)}$.

Os conflitos surgem entre as várias categorias profissionais e são comuns os desentendimentos entre classe médica e de enfermagem. Estes irão interferir no andamento do trabalho, prejudicando o desempenho e motivação dos profissionais envolvidos e sucessivamente na qualidade da assistência prestada colocando em risco a segurança do paciente. É um dos principais problemas nos serviços de saúde e podem ocasionar problemas éticos afetando de forma negativa a relação ${ }^{(10)}$.

Vários são os fatores geradores de conflitos nos serviços de saúde e entre eles podem ser citados a limitação dos recursos, as condições de trabalho, a falta de definição do espaço de atuação profissional, as tecnologias utilizadas no processo de trabalho e o alto nível de exigência das instituições e dos pacientes em relação ao trabalho desenvolvido pelos profissionais ${ }^{(11)}$.

A comunicação é considerada um fator de segregação ou agregação dependendo do modo como ocorrerá e nas relações entre os profissionais de saúde os problemas são constantes e irão interferir diretamente na continuidade e qualidade do serviço ${ }^{(12)}$. Portanto, é um elemento essencial quando se refere ao relacionamento interpessoal e necessária para o alcance das metas do cuidado. É através dela que o profissional expressa suas opiniões e sentimentos dentro do local de trabalho. Ela faz parte do processo do cuidar em enfermagem, pois fortalece a interação entre as equipes, pacientes, familiares e deve ser conduzida com ética com a intenção de harmonizar as relações humanas ${ }^{(13)}$.

É necessário compreender que se a comunicação entre os membros da equipe for ineficaz poderá surgir fatores causadores de insatisfação nas instituições de saúde, ocasionando uma assistência precária, sujeito a iatrogenias ${ }^{(13)}$. O estudo teve como questão norteadora e objeto as relações entre as equipes de enfermagem e médica no Centro Cirúrgico e como objetivo desvelar sentidos da equipe de enfermagem em centro cirúrgico nas suas relações interpessoais com a equipe médica.

\section{MÉTODOS}

Pesquisa qualitativa sustentada no
referencial teórico, filosófico e metódico da 
fenomenologia, pois esta permite imergir na subjetividade, ir às coisas que se apresentam encobertas e compreender o fenômeno, pois cada pessoa é ímpar e uma mesma situação pode ser vivenciada de modo diferente por cada pessoa $^{(14)}$. Assim, a fenomenologia é o estudo dos fenômenos, é deixar e fazer ver por si mesmo aquilo que se mostra, tal como se mostra a partir de si mesmo ${ }^{(15)}$.

O cenário do estudo foi o Centro Cirúrgico de um Hospital Universitário, situado em um município na Zona da Mata Mineira. Os participantes foram enfermeiros e técnicos de enfermagem que integram a equipe do setor, perfazendo um total de dezessete profissionais.

As entrevistas foram realizadas entre os meses de outubro de 2016 a janeiro de 2017, no próprio local de trabalho dos participantes a pedido dos mesmos sem, no entanto, interferir no andamento do serviço, em horários previamente agendados, em uma sala que garantiu a privacidade destes. O convite se deu de modo individual e nesse momento foi esclarecido o objetivo da pesquisa, assim como a possibilidade de desistência sem qualquer ônus.

Como critérios de inclusão foram considerados os profissionais de enfermagem, ambos os sexos e que possuíam no mínimo seis meses de experiência no setor e os critérios de exclusão foram os profissionais que se encontravam de folga, férias ou licença no momento da entrevista.

Os depoimentos foram coletados por meio de entrevista aberta com o uso de um gravador digital. O tempo médio de entrevista foi de 10 minutos sendo utilizada à questão norteadora: Como você significa as suas relações interpessoais no seu setor de trabalho. Após transcrição integral do conteúdo, as falas foram transcritas na íntegra e analisadas repetidas vezes buscando assim fidelidade das informações. Valeu-se do diário de campo a fim de registrar as expressões relacionadas à linguagem não verbal. Utilizada a técnica de marcação com cores delimitando as falas que se aproximavam do objeto em estudo. $\mathrm{Na}$ análise, as informações foram organizadas a fim de agrupar os relatos que se apresentavam semelhantes e a partir desse agrupamento emergiram as estruturas essenciais que possibilitaram a constituição da unidade de significação ${ }^{(15)}$.

Os participantes receberam como pseudônimos nomes de elementos percepcionais e afetivos do cotidiano visto que no setor são vivenciados diariamente inúmeros sentimentos e emoções e tais sentimentos os acompanham com intensidade. A escolha do pseudônimo foi realizada pela pesquisadora após o encontro fenomenológico de acordo com o sentimento ou emoção que o depoente expôs no decorrer do seu depoimento.

O estudo em questão teve seu projeto de pesquisa aprovado pelo Comitê de Ética em Pesquisa com Seres Humanos da Universidade Federal de Juiz de Fora sob parecer no. 1.049.543/2015.

\section{RESULTADOS E DISCUSSÃO}

A idade dos participantes variou entre 29 e 63 anos. Com relação à ocupação, três profissionais atuavam como enfermeiros, doze como técnicos de enfermagem e dois como auxiliares. Dos dezessete entrevistados, onze possuíam curso superior. $O$ tempo de atuação no setor variou de 06 meses a 26 anos.

A partir das estruturas essenciais foi organizada a Unidade de Significação que compreende o primeiro momento metódico, a compreensão vaga e mediana. Assim, os profissionais da equipe de enfermagem em centro cirúrgico significaram que as relações interpessoais com a equipe médica são boas, mas têm conflitos e divergências.

A equipe de enfermagem relatou que a relação com os profissionais médicos é boa e tranquila:

"Tenho um bom relacionamento interpessoal (Amor). Cada um sabe o seu momento e a gente trabalha tranquilamente." (Confiança)

"Vejo uma relação muito boa, não tenho dificuldade com isso não."(Tolerância)

"Todo mundo tem que estar sempre trabalhando junto ali um pelo outro. Se não tiver essa equipe, o trabalho não flui legal (Tranquilidade). Um ajuda o outro e isso que é importante." (Sensatez)Divergências e conflitos se fazem presentes como pode ser observado nas falas:

Posso ter divergência com o colega, mas o profissionalismo não pode interferir." (Respeito)

"Tem as divergências que tem em todo lugar mais não é nada grave, nada que não dê pra ser contornado e é isso." (Carinho)

"São uns pequenos conflitos, que não é tudo perfeito lógico."(Tolerância)

"Boa parte dos conflitos ainda tem acontecido por inexperiência mesmo." (Carisma) 
"Eles estressam com qualquer coisa, mas quer empurrar a culpa pra cima da enfermagem." (Empatia)

Evitam conflitos e preferem não falar ou esperar o momento de estresse passar:

"A gente não dá muita confiança...ele fala, eu ouço, eu não bato boca com ninguém nunca, não vou discutir, eles falam, eu ouço."(Tolerância)

"Se a pessoa está estressada na hora, aí eu deixo passar aquele momento de estresse aí eu converso com a pessoa quando ela está tranquila." (Paciência)

"Eu prefiro não falar, eu prefiro evitar atritos." (Paixão)

"No serviço mesmo, mais conversa, essas coisas mesmo com os médicos, quem faz mais são as enfermeiras." (Harmonia)

Por vezes ocorre uma falta de consideração pela opinião e pelo conhecimento do profissional assim como uma falta de respeito e o não reconhecimento de seu trabalho:

"A gente sempre se impõe porque senão $o$ paciente acaba sendo o maior prejudicado. Você sabe que o médico acha que é o dono de tudo." (Confiança)

"A gente observa que essa parte que teoricamente a equipe de enfermagem entende como o básico, como crucial pra garantir a segurança do paciente, nem sempre é levado em consideração pela equipe médica." (Carisma)

"A maior parte deles (médicos) não olha pra gente como profissional, olha pra gente como, qualquer pessoa faz isso daí, ou se você não tiver na sala não faz diferença." (Equilíbrio)

"Às vezes eles conseguem fazer com que eu me sinta muito inferior a eles, um nada, um zero a esquerda. Ninguém leva em consideração o que você fala." (Paixão)

"Existe uma minoria meio babaca que as vezes gosta até de menosprezar o serviço da gente, acha que o serviço da gente é uma coisa assim, que qualquer um pode fazer." (Serenidade)

"Que eles sempre menosprezam a gente. Você pode fazer o melhor possível que nunca está bom, parece que já se habituou a desfazer do seu serviço." (Empatia)

O mostrar-se da equipe de enfermagem acerca de suas relações interpessoais no Centro Cirúrgico a partir dos significados estabelecidos por ela foi apontado na compreensão vaga e mediana ou primeiro momento metódico emergindo as unidades de significação sendo então construído o conceito de ser, que é o fio condutor da hermenêutica. Desse modo, a partir do fio condutor caminha-se da dimensão ôntica à ontológica, que constituirá o segundo momento metódico em Heidegger, a compreensão interpretativa ou hermenêutica. Para constituir o ser é necessário ir além do ente.

Nas relações interpessoais estabelecidas no setor, o ser-aí se encontrou lançado no mundo e se confrontou com a facticidade pertencente a esse mundo e desse modo ser-aí é obrigatoriamente ser-no-mundo-com-os-outros. O mundo do ser-aí é sempre mundo-com, no qual o ser-em é ser-com-os-outros se constituindo como imprescindível do existir humano podendo ocorrer de vários modos, não sendo conferido valor positivo ou negativo aos diferentes modos de sermos-uns-com-os-outros. Podemos ser unscom-os-outros amando ou odiando, cuidando ou descuidando, capacitando ou incapacitando ${ }^{(16)}$.

$O$ outro é aquele que está com o ente que eu mesmo sou em um mundo comum. Ao mesmo tempo em que o ser-no-mundo se torna ser-comos-outros, o próprio mundo se estabelece como mundo com-partilhado. O outro não é apenas o outro, é um co-existente. Deste modo, na relação com o outro Dasein percebo ao meu próprio Dasein seu caráter de ser-com ${ }^{(17)}$. O ser humano está sempre se relacionando com aqueles que o cercam, mesmo que de modo incompleto, pois o ser-aí é fundamentalmente ser-com. Faz parte da condição do ente ser-com ao ser-no-mundo, por isso o mundo é sempre com-partilhado ${ }^{(15)}$.

Os depoentes desvelaram-se na impropriedade ao tentarem evitar os conflitos não falando o que pensavam, preferiram se calar ao invés de falar atingindo assim o modo da inautenticidade. Há dois modos de estar-nomundo: como existência autêntica ou modo próprio de ser e como existência inautêntica ou modo impróprio de ser. A existência autêntica trata-se da propriedade do ser onde o ser-aí se reconhece como presença deixando o anonimato e a impessoalidade. Na existência inautêntica o ser-aí se encontra na impropriedade sem uma direção que lhe é própria ${ }^{(15)}$.

Embora o homem seja um ente de existência, na maior parte do tempo essa existência é inautêntica, pois no cotidiano ele está entregue ao falatório e às opiniões que vêm dos outros o que resulta no encobrimento de seu ser ou em numa compreensão errônea do seu próprio existir. Assim, a inautenticidade ocorre quando deixamos de possuir nós mesmos ou não 
nos responsabilizamos por nossos próprios desejos $^{(18)}$.

A ambiguidade foi desvelada no momento em que os membros da equipe relataram que a relação tanto entre eles quanto entre a equipe médica era boa, a enfermagem era respeitada e não havia dificuldades de relacionamento. Entretanto afirmaram que apesar de ser uma relação tranquila, os conflitos e as divergências se faziam presentes e tratavam bem quando foram tratados bem.

Houve falta de comunicação entre os profissionais, o não pedir ajuda a outro profissional que dividia o mesmo espaço por não estarem se falando, mas que necessitava ajuda, o não querer determinado profissional auxiliando na cirurgia, o não levar em consideração o que o outro falava, desvelou o distanciamento. Somente ao se descobrir para a presença a distância dos entes é que no próprio ente intramundano tornam-se acessíveis distanciamentos e intervalos com referência a outros entes ${ }^{(15)}$.

Os participantes partiram da facticidade de trabalhar nesse Centro Cirúrgico. Desvelaram-se na impropriedade ao evitarem conflitos não falando o que pensavam atingindo assim o modo da inautenticidade. Atingiram o modo da ambiguidade ao relatarem que o relacionamento era muito bom quando na verdade não era, mas também afirmaram que o relacionamento não era bom quando permaneceram trabalhando e se relacionando com toda a equipe de maneira educada e satisfatória. Distanciaram-se quando ocuparam o mesmo espaço geográfico, mas não se falavam.

As relações interpessoais podem afetar de forma positiva ou negativa o desenvolvimento do trabalho e a satisfação profissional como observado em alguns depoimentos. A insatisfação, a desvalorização profissional e a comunicação foram alguns dos problemas evidenciados pelos participantes e que irão influenciar diretamente a qualidade do serviço prestado, corroborando com alguns estudos ${ }^{(8,10-}$ 13)

Não há interação sem comunicação e ambas são fundamentais para o cuidado(12). A comunicação deficiente contribui para um ambiente estressante. Assim, ela pode ser considerada como uma ferramenta essencial no ambiente de trabalho pois é através dela que o profissional consegue se expressar fortalecendo assim as relações interpessoais ${ }^{(7,13)}$
Para que a comunicação seja otimizada é necessário desenvolver algumas estratégias a fim de minimizar os problemas e fortalecer as relações interpessoais. Reuniões periódicas podem surgir como uma estratégia de aproximação dos profissionais e que irão influenciar o relacionamento interpessoal das equipes, com o objetivo de aprimorar as habilidades comunicativas gerenciais e, assim criar competências para lidar melhor com os profissionais $^{(12)}$.

A prática multiprofissional se mostra indispensável no contexto do trabalho mas ao mesmo tempo é considerada um desafio na medida em que é necessário reconhecer o outro e a importância do seu trabalho ${ }^{(7)}$. Desse modo, palestras motivacionais e dinâmicas de grupo também poderiam ser consideradas estratégias onde cada profissional possa compreender seu papel e o papel desempenhado pelo outro.

A fragmentação na saúde reforça o isolamento profissional e um maior empenho pelo reconhecimento profissional, entretanto não se pode diminuir a capacidade de a equipe voltar seu olhar para o outro, e reconhecer a importância de todos os envolvidos para o resultado final, a assistência de qualidade ${ }^{(7)}$.

\section{CONSIDERAÇÕES FINAIS}

A partir da compreensão dos significados expressos nos depoimentos dos participantes foi possível desvelar sentidos da equipe de enfermagem em centro cirúrgico nas suas relações interpessoais com a equipe médica. 0 caminhar investigativo apontou as relações interpessoais como sendo fundamentais para o desenvolvimento das atividades do setor, pois favorece a qualidade da assistência. Entretanto em alguns momentos essas relações mostraramse conflituosas o que pode estar relacionado com as peculiaridades do setor.

Observa-se que a comunicação é imprescindível para a realização do processo de trabalho e um desafio constante entre as equipes, principalmente no Centro Cirúrgico por se tratar de um setor fechado com inúmeras situações complexas envolvendo os profissionais que enfrentam uma rotina intensa e estressante devido aos procedimentos específicos que ali ocorrem, as intercorrências que surgem ao longo do dia de trabalho e a proximidade em que se encontram favorecendo as tensões e os conflitos.

O ser-com a equipe possibilita o exercício da empatia na medida em que o profissional se 
encontra aberto ao diálogo e à escuta, pois promove a troca de conhecimentos e como consequência à assistência adequada ao paciente. Identificou-se por meio dos resultados a adoção da prevenção do estresse ocupacional visando o ambiente de trabalho de trabalho e a assistência de qualidade ao paciente cirúrgico.

Compreende-se como limitação deste estudo a realização ter ocorrido em somente uma unidade de centro cirúrgico. Mas, acredita-se que mesmo assim trouxe contribuições para reflexões e incentivo ao desenvolvimento de outros para suprir a lacuna da temática na literatura.

Outra limitação se refere às publicações atuais sobre a temática específica das relações interpessoais em centro cirúrgico. Ademais sugere-se novos estudos dessas relações voltadas para a temática de centro cirúrgico pois estudos atuais são escassos, porém essenciais para uma melhor compreensão dessas relações.

\section{REFERÊNCIAS}

1. Martins FZ, Dall'Agnol CM. Surgical center: Challenges and strategies for nurses in managerial activities. Ver Gaúcha Enferm. 2016;37(4):1-9. DOI: 10.1590/1983$\underline{1447.2016 .04 .56945}$

2. Medeiros A, Araújo-Filho I. Centro cirúrgico e cirurgia segura. J Surg $\mathrm{Cl}$ Res 2017;8(1):77-105. DOI: 10.20398/jscr.v8i1.13037

3. Associação Brasileira de Enfermeiros em Centro Cirúrgico, Recuperação Pós-Anestésica e Centro de Material e Esterilização (SOBECC). Esterilização de produtos para a saúde. In: Associação Brasileira de Enfermeiros em Centro Cirúrgico, Recuperação Pós-Anestésica e Centro de Material e Esterilização (SOBECC). Diretrizes de práticas em enfermagem cirúrgica e processamento de produtos para a saúde. Sa?o Paulo: SOBECC; 2017.

4. Rachwal CM, Langer T, Trainor LT, Bell MA, Browning DM, Meyer EC. Navigating Communication Challenges in Clinical Practice: $\mathrm{A}$ new approach to team education. Crit Care Nurse 2018;38(6):15-22. DOI: $10.4037 / \operatorname{ccn} 2018748$

5. Abreu TFK, Amendola F, Trovo MM. Relational technologies as instruments of care in the Family Health Strategy. Ver Bras Enferm. 2017;70(5):981-7. DOI: 10.1590/0034-7167-20160337

6. Araújo MPS, Medeiros SM, Quental LLC. Interpersonal relations among nursing staf: Fragilities and strengths. Rev Enferm UERJ 2016; 24(5):1-5. DOI: 10.12957/reueri.2016.7657
7. Trajano MFC, Gontijo DT, Silva MW, Aquino JM, Monteiro EMLM. Interpersonal relationships in the surgical unit from the perspective of nursing workers: An exploratory study. Braz J Nurs. 2017 [citado em 12 out 2019]; 16(1):159-69. Disponível em: http://www.objnursing.uff.br/index.php/nursing/ article/view/5530

8. Barros ERS, Ellery AEL. Inter-professional collaboration in an Intensive Care Unit: Challenges and opportunities. Rev Rene 2016;17(1):10-9.

DOI: 10.15253/rev\%20rene.v17i1.2600

9. Kwiecie?-Jagu? K, M?drzycka-D?browska W, Chamienia A, Kielaite V. Stress factors vs. job satisfaction among nursing staff in the Pomeranian Province (Poland) and the Vilnius Region (Lithuania). Ann Agric Environ Med. 2018;25(4):616-24. DOI: 10.26444/aaem/75801

10. Amestoy SC, BackesVMS, Thofehrn MB, Martini JG, Meirelles BHS, Trindade LL. Con?ict management: Challenges experienced by nurseleaders in the hospital environment. Rev Gaúcha Enferm. 2014;35(2):79-85. DOI: 10.1590/19831447.2014.02.40155

11. Eduardo EA, Peres AM, Kalinowski CE, Cunha ICKO, Bernardino $E$. The negotiator that we have and the negotiator that we want in nursing. Texto Contexto - Enferm. 2016;25(3):1-9. DOI: 10.1590/0104-07072016001030015

12. Broca PV, Ferreira MA. Communication process in the nursing team based on the dialogue between Berlo and King. Esc Anna Nery 2015;19(3):467-74. DOI: 10.5935/14148145.20150062

13. Martins CCF, Santos VEP, Pereira MS, Santos NP. The nursing team's interpersonal relationships v. stress: Limitations for practice. Cogitare Enferm. 2014;19(2):287-93. DOI: 10.5380/ce. v19i2.36985

14. Silva AL, Silva LF, Souza IEO, Moreira RVO. Female patient that suffers from heart disease with pressure ulcer: A phenomenological reflection on a clinical care model of comfort. Esc Anna Nery 2013; 17(1):168-72. DOI: 10.1590/S1414-81452013000100023

15. Heidegger M. Ser e tempo. Petrópolis: Vozes; 2015.

16. Santos AG, Monteiro CFS, Nunes BMVT, Benício CDAV, Nogueira LT. The nursing care analyzed according the essence of the care of Martin Heidegger. Rev Cubana Enferm. 2017 [citado em 23 set 2018]; 33(3). Disponível em: 
http://www.scielo.br/pdf/jbn/v38n3/0101-2800jbn-38-03-0313.pdf

17. Braga TBM, Farinha MG. Heidegger: Searching for sense for the human existence. Rev Abordagem Gestált 2017 [citado em 23 set 2018]; 23(1):65-73. Disponível em: http://pepsic.bvsalud.org/scielo.php?script=sci a rttext\&pid=S1809-68672017000100008

18. Santos R, Mohr AM. A (de) vida angústia de morte: Considerações a partir da filosofia e da psicanálise. Nat Hum 2018 [citado em 23 set 2018]; 20(1):169-87. Disponível em: http://revistas.dwwe.com.br/index.php/NH/articl e/view/318/210

Nota: Manuscrito baseado na dissertação "Significados e Sentidos das relações Interpessoais dos Profissionais de Enfermagem em Centro Cirúrgico" apresentada ao PPG da Faculdade de Enfermagem da Universidade Federal de Juiz de Fora - MG.

Recebido em: 29/03/2019

Aprovado em: 23/10/2019

Endereço de correspondência:

Anna Maria de Oliveira Salimena

Rua Marechal Cordeiro de Farias, 172.

CEP: 36081-330 - Juiz de Fora/MG - Brasil

E- mail: annasalimena@terra.com.br 